\title{
Revisión documental de factores de producción analizados en investigaciones del sector metalmecánico en Colombia durante 2015-2019
}

\section{Documentary Review of Production Factors Analyzed in Investigations of the Metalworking Sector in Colombia During 2015-2019}

César Augusto Figueredo Garzón ${ }^{1}$

Nidia Estela Rincón Parra ${ }^{2}$

Hilda Lucia Jiménez Orozco ${ }^{3}$

Flor Marlen Ávila Guerrero ${ }^{4}$

\section{OPEN ACCESS}

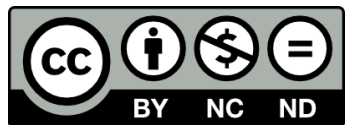

\section{Copyright:}

(02020. La revista Ingenierías USBmed proporciona acceso abierto a todos sus contenidos bajo los términos de la licencia creative commons Atribución no comercial SinDerivar 4.0 Internacional (CC BY-NC-ND 4.0)

Tipo de artículo: Revisión.

Recibido: 22-08-2019.

Revisado: 06-05-2020.

Aprobado: 26-05-2020.

Doi: $10.21500 / 20275846.4249$

\section{Referenciar así:}

C. A. Figueredo-Garzón, N. E. RincónParra, H.L. Jimenez-Orozco y F.M. Ávila-Guerrero, "Revisión documental de factores de producción analizados en investigaciones del sector metalmecánico en Colombia durante 2015-2019," Ingenierías USBMed, vol. 11, no. 2, pp. 54-61, 2020.

\section{Disponibilidad de datos:}

todos los datos relevantes están dentro del artículo, así como los archivos de soporte de información.

\section{Conflicto de intereses:}

los autores han declarado que no hay conflicto de intereses.

Editor: Andrés Felipe Hernández. Universidad de San Buenaventura, Medellín, Colombia.

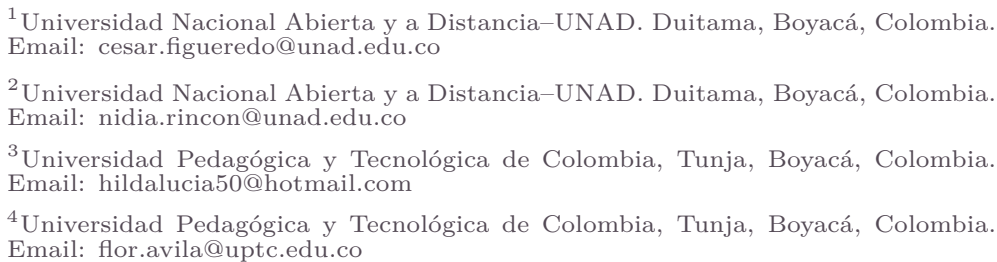

Resumen. El sector metalmecánico colombiano enfrenta brechas tecnológicas en automatización industrial, restricciones como altas cargas impositivas, dificultad para el acceder al apalancamiento financiero, falencias en gestión, en planes tecnológicos y análisis de oferta y demanda. En concordancia, es necesario conocer el sector a partir del cuestionamiento: £cómo contextualizar el sector metalmecánico de Colombia y poder conocer los factores de producción objeto de mayor análisis? El objetivo es realizar una revisión y análisis documental exploratorio, siendo objeto de investigación 50 artículos entre 2015 y 2019 del sector. El estudio se hizo con la matriz de análisis de investigaciones, identificando factores comunes, preponderantes y significativos en los estudios seleccionados, concluyendo que el sector está en un entorno de alta competitividad si desea obtener permanencia y evolución. La dinámica incluye compresión de factores: productividad, producción, competitividad, niveles tecnológicos, oportunidades del sector y talento humano; siendo los de producción objeto de mayor análisis en los estudios utilizados. Esto permite contextualizarlo y entenderlo; inferir la utilización de estrategias como disminución de costos, gestión de calidad, aplicación de forma valuada y significativa de la acumulación de experiencia, la construcción de cadenas de valor en torno a estructuras de trabajo mancomunado y la identificación de la brecha tecnológica.

Palabras Clave. Productividad, competitividad, personal, tecnología avanzada, mercado, exportaciones, producción, sector industrial.

Abstract. The Colombian metalworking sector faces technological gaps in industrial automation, restrictions such as high tax burdens, difficulty in accessing financial leverage, management failures, in technological plans and analysis of supply and demand. Accordingly, it is necessary to know the sector, based on the questioning: How to contextualize the metalworking sector of Colombia and be able to know the factors of production that are the object of further analysis? The objective is to carry out an exploratory documentary review and analysis, with 50 articles being investigated between 2015 and 2019 in the sector. The study was done with the research analysis matrix, identifying common, predominant and significant factors in the selected studies; concluding that the sector is in a highly competitive environment if it wishes to obtain permanence and evolution. The dynamics include compression of factors: productivity, production, competitiveness, technological levels, sector opportunities and human talent; being those of production object of greater analysis in the used studies. This allows to contextualize it and understand it; inferring the use of strategies such as cost reduction, quality management, valued and significant application of the accumulation of experience, the construction of value chains around joint work structures, and the identification of the technological gap.

Keywords. Productivity, Competitiveness, Personnel, Advanced Technology, Market, Exports, Production, Industrial Sector. 


\section{Introducción}

El sector metalmecánico en Colombia se ha convertido en uno de los renglones económicos con mayor proyección. Sin embargo, debe enfrentar dificultades y brechas a diferentes niveles, siendo la competitividad un factor fundamental en el quehacer de este sector y de su relación con los otros sectores productivos. Se tiene identificado que la producción total de las cinco siderúrgicas más grandes de Colombia presentaron una reducción del $9 \%$ en su producción total al final del año anterior debido a las importaciones provenientes de Turquía y China. También se debe tener en cuenta que hay competidores con una fuerte influencia: Corea del Sur, China, India y Tailandia [1]. El sector metalmecánico se está enfrentando a las brechas tecnológicas en automatización industrial, restricciones externas como las altas cargas impositivas, los impuestos y una gran dificultad para el acceso a fuentes de apalancamiento financiero, así como falencias en la gestión y planes tecnológicos. Además, no se tienen identificadas de manera clara las necesidades del mercado.

Para [2] los productores nacionales deben propender por el uso óptimo de los recursos, flexibilizar el sistema productivo, realizar entregas oportunas y buscar un manejo responsable para la protección del medio ambiente, con el fin de buscar armonía en toda su producción y competitividad en los mercados internacionales.

En concordancia con lo anterior se propone realizar una revisión documental de los factores de producción analizados en investigaciones del sector metalmecánico en Colombia en el periodo 2015-2019, para contextualizar y comprender el sector y sus diversas alternativas de estudio, logrando consolidar un cimiento académico que sirva de fundamento en las acciones estratégicas para el sector.

Para lograr lo anterior es necesario, en primera instancia, realizar la recolección de la información a través de un instrumento en Excel que permita identificar los artículos útiles para el objeto de estudio, utilizando como primer filtro los estudios del sector metalmecánico en el periodo 2015-2019. En segunda instancia, realizar la evaluación de la información a través de la matriz de análisis de investigaciones, para depurar y contextualizar el sector a partir de la identificación de los factores comunes de los estudios seleccionados. Y finalmente llegar a estructurar el cimiento académico a partir de la comprensión de la situación actual y de cómo se está abordando el sector, con el fin de poder plasmar su dinámica organizacional.

En busca de lo planteado anteriormente se parte de la pregunta: ¿cómo contextualizar el sector metalmecánico de Colombia y poder conocer los factores de producción objeto de mayor análisis en estudios realizados?
El estudio se justifica porque es necesario comprender y analizar aquellos factores de producción que permitan fortalecer las acciones provechosas que contribuyan a la reducción de brechas identificadas y contrarresten las dificultades encontradas en el análisis realizado, para así constituir lineamientos y disposiciones encaminadas a propender por prioridades competitivas y de sostenibilidad del sector sin dejar de lado la búsqueda del equilibrio organizacional, que incluye la responsabilidad social y medioambiental.

\section{Metodología}

Se realizó una revisión y análisis documental exploratoria a través de fuentes de información secundarias, donde el objeto de investigación fueron 50 artículos entre el periodo 2015-2019 sobre el sector metalmecánico en Colombia, para poder entender, explicar y analizar el contexto del sector. "Esta es la industria que se encarga de transformar el acero en bienes que van desde laminados, tuberías, estructuras metálicas y alambres, hasta maquinaria industrial como ascensores y calderas. Actualmente existen más de 680 empresas dedicadas al sector metalmecánico a lo largo de la cadena manufacturera" [3].

Para la recolección de la información se diseñó un instrumento en Excel que tiene como elementos de selección: título, año, autores, resumen y variables utilizadas. Dichos artículos se tomaron de bases de datos y portales bibliográficos que cuentan con respaldo académico. Se registraron todos los artículos utilizando como filtros iniciales: sector metalmecánico en Colombia y el intervalo de tiempo 2015-2019; de allí se extrajeron los 50 artículos seleccionados. De acuerdo a lo exigido para artículos de reseña o revisión, los cuales contienen información fidedigna y relevante para los fines del estudio, las categorías de análisis fueron los factores de producción comunes de mayor relevancia en los artículos objeto de estudio, lo que permiten comprender la dinámica del sector. Dichos factores encontrados fueron: producción y productividad; competitividad, niveles tecnológicos, oportunidad del sector y talento humano.

La unidad de análisis la constituye cada una de las investigaciones contempladas en el presente estudio.

Se procedió a realizar el estudio-evaluación de la información. Asimismo, se utilizó una matriz de análisis de investigaciones, donde las categorías establecidas son el resultado o "proceso mediante el cual se agrupa la información obtenida en categorías que concentran las ideas, conceptos o temas similares descubiertos por el investigador" [4].

\section{Resultados}

La Tabla 1 está constituida por las fuentes documentales tomadas como objeto de estudio para identificar los factores de producción más analizados, esto con el fin de contextualizar y comprender el sector metalmecánico en Colombia. 
Tabla 1. Estudios analizados presentes en la investigación.

\begin{tabular}{|c|c|}
\hline No. & Título del artículo \\
\hline 1 & $\begin{array}{l}\text { Sector Metalmecánico del Departamento de } \\
\text { Santander en El Periodo } 20102015 \text {. [5] }\end{array}$ \\
\hline 2 & $\begin{array}{l}\text { Niveles tecnológicos de las empresas del sec- } \\
\text { tor metalmecánico en Boyacá desde el dis- } \\
\text { eño y manufactura asistida por computador } \\
(\mathrm{CAD} / \mathrm{CAM}) \text {. [6] }\end{array}$ \\
\hline 3 & $\begin{array}{l}\text { La innovación tecnológica: mecanismo de com- } \\
\text { petitividad para la creación de un clúster en } \\
\text { el sector metalmecánico de los municipios de } \\
\text { Cali y Yumbo. [7] }\end{array}$ \\
\hline 4 & $\begin{array}{l}\text { Perfil innovador de la industria manufacturera } \\
\text { colombiana. Caso del sector Metalmecánico } \\
\text { de Barranquilla. [8] }\end{array}$ \\
\hline 5 & $\begin{array}{l}\text { Sector Metalmecánico de Risaralda un Dia- } \\
\text { mante listo para ser pulido. }[9]\end{array}$ \\
\hline 6 & Valoración de la Empresa Técnica \\
\hline & $\begin{array}{l}\text { Metalmecánica del Caribe y Cía. Ltda. } \\
\text { Una empresa del sector Metalmecánico de la } \\
\text { Ciudad de Cartagena. [10] }\end{array}$ \\
\hline 7 & $\begin{array}{l}\text { Propuesta de implementación de sistema ge- } \\
\text { neral de mantenimiento basado en manteni- } \\
\text { miento productivo total y confiabilidad en el } \\
\text { sector metalmecánico. [11] }\end{array}$ \\
\hline 8 & $\begin{array}{l}\text { Propuesta de mejoramiento de la productivi- } \\
\text { dad de una Pyme del sector metalmecánico } \\
\text { de estructuras en Bogotá como estrategia } \\
\text { para competir contra las importaciones de } \\
\text { China. [12] }\end{array}$ \\
\hline 9 & $\begin{array}{l}\text { Estudio prospectivo para el sector } \\
\text { metalmecánico en Boyacá. [13] }\end{array}$ \\
\hline 10 & $\begin{array}{l}\text { Carga Física en Trabajadores del Área de } \\
\text { Acabados en Industria Metalmecánica. [14] }\end{array}$ \\
\hline 11 & $\begin{array}{l}\text { Prospectiva Laboral para el Sector } \\
\text { Metalmecánico en Boyacá. }[15]\end{array}$ \\
\hline 12 & $\begin{array}{l}\text { Determinación de brechas estructurales en la } \\
\text { integración de la Responsabilidad Social en } \\
\text { empresas de la región Centro-Sur de Caldas. } \\
\text { Caso de Estudio: Medianas empresas del sec- } \\
\text { tor metalmecánico. }[16]\end{array}$ \\
\hline 13 & $\begin{array}{l}\text { Metodología para implementar las } 5 \text { 's en em- } \\
\text { presas del sector metalmecánico del corredor } \\
\text { industrial de Boyacá. [17] }\end{array}$ \\
\hline 14 & $\begin{array}{l}\text { Diagnóstico del nivel de capacitación del } \\
\text { personal vinculado al sector siderúrgico y } \\
\text { metalmecánico de Boyacá respecto a las nor- } \\
\text { mas de competencia laboral. [18] }\end{array}$ \\
\hline 15 & $\begin{array}{l}\text { Dinámica de la cadena priorizada del sec- } \\
\text { tor metalmecánico del departamento de San- } \\
\text { tander en el período } 2010-2015 \text {. [19] }\end{array}$ \\
\hline 16 & $\begin{array}{l}\text { La industria metalmecánica en Colombia } \\
\text { frente a los TLC. [20] }\end{array}$ \\
\hline
\end{tabular}

17 Factores de calidad que afectan la productividad y competitividad de las micros, pequeñas y medianas empresas del sector industrial metalmecánico. [21]

18 Diseño de un modelo para la implementación de procesos de innovación aplicables a la industria en empresas metalmecánicas en BOGOTÁ. [22]

19 Una mirada retro y prospectiva en las Pymes del sector metalmecánico de Bogotá. [23]

20 Sector autopartes en Colombia. [24]

21 Una mirada retro y prospectiva en las Pymes del sector metalmecánico de Bogotá. [25]

22 Diseño del modelo de planeación de operaciones de compras e inventarios aplicado al sector metalmecánico de la ciudad de CARTAGENA. [26]

23 Elementos determinantes del proceso de desarrollo de nuevos productos en cinco pymes metalmecánicas: método hermenéutico fenomenológico. [27]

24 Comercio intrarregional de los sectores autopartes y metalmecánico del departamento de Santander con los países miembro de la Alianza del Pacífico en el periodo 2012-2015. [28]

25 Industria metalmecánica, a competir con sostenibilidad. [29]

26 Análisis estratégico a partir del modelo de prácticas de gestión verde en las empresas industriales del sector metalmecánico de Manizales, Caldas Colombia. [30]

27 Relaciones Comerciales entre los Países Miembros de la Alianza Del Pacífico En El Sector Metalmecánico. [31]

28 Inversión extranjera directa en el sector metalmecánica de Risaralda Colombia. [32]

29 Diseño de un plan de gestión integral de residuos sólidos para una industria metalmecánica en la localidad de Puente Aranda. [33]

30 Bienes de Capital desde el Caribe Colombiano, la historia de SUPERBRIX. [34]

31 Aplicación de herramientas Lean Manufacturing en los procesos de recepción y despacho de la empresa HLF Romero S.A.S. [35]

32 Escenarios futuros del sector metalmecánico. Municipio de Tuluá y su zona de influencia. Horizonte 2018-2028. [36]

33 Estrategias Lean Manufacturing para una empresa del sector metalmecánico. [37]

34 Desarrollo De Estrategias De Manufactura Sostenible En La Micro, Pequeña Y Mediana Empresa Metal Mecánica (MIPYMES). [38]

35 Diversificación Inteligente: Posibilidades de diversificación y sofisticación de la Industria Metalmecánica en Colombia. [39] 
36 Modelo para el diagnóstico de madurez en la Gestión de Proyectos para Mipymes de mantenimiento del sector metalmecánico en la localidad de KENNEDY. [40]

37 Análisis de los canales de distribución de las MIPYMES del sector metalmecánico de la ciudad de Barranquilla para establecer un modelo de negocio en el sector. [41]

38 Impacto de los indicadores del modelo SCOR para el mejoramiento de la cadena de suministro de una siderúrgica, basados en el ciclo cash to cash. [42]

39 Incidencia diferencial de los regímenes laborales Mipyme y común en el estado de resultados de las empresas del sector metalmecánico de la ciudad de Cajamarca para el año 2017. [43]

40 Oportunidades que la alianza del pacífico crea en beneficio del sector metalmecánico a la competitividad de las pymes del municipio de Dosquebradas. [44]

41 Enfoque competitivo-Negocio Metalmecánico. [45]

42 Sector metalmecánico, uno de los más grandes del país: Fedemetal. [46]

43 Diseño de arquitectura de negocio basado en capacidades de tecnologías de la información para pyme del sector metalmecánico. [47]

44 Criterios de implementación ISO 14001:2015 estudio de caso FUTECO sector metalmecánico. [48]

45 Estudio de Caracterización de las Áreas de Mantenimiento en Empresas del Sector Metalmecánico En Bogotá Y Sus Alrededores. [49]

46 Tendencia de la cultura organizacional en las pymes metalmecánicas en la localidad de Fontibón, Bogotá D.C. [50]

47 Rediseño del Sistema de Gestión de la Calidad a través de metodologías de mejora de procesos para incrementar la productividad en una empresa metalmecánica de Bogotá, [51]

48 Aspectos del plan de prevención, preparación y respuestas ante emergencias en el Sistema de Gestión de la Seguridad y Salud en el Trabajo. [52]

49 Evaluación de la Cadena de Suministro para Mejorar la Competitividad y Productividad en el Sector Metalmecánico en Barranquilla, Colombia. [53]

50 Metodología de distribución de plantas en ambientes de agrupación celular. [54]

En la Tabla 2 se presentan los resultados del estudioevaluación de la información utilizando una matriz de análisis de investigaciones a partir de los factores que más se concentran y que aportan información descriptiva y significativa en las investigaciones objeto de estudio.

De acuerdo a los resultados obtenidos los factores objeto de producción que están siendo analizados de forma más preponderante son: producción y productividad, competitividad, niveles tecnológicos, oportu- nidades del sector y talento humano. Para poder realizar su análisis contextual es necesario hacer una comprensión epistemológica de cada uno de ellos, partiendo de enfoques comunes que articulen dichos factores a la necesidad de conocer cómo se está desempeñando el sector desde su competitividad, que es estimada como el asiento fidedigno para el desarrollo de un país.

Tabla 2. Consolidación de resultados obtenidos en matriz de análisis de investigaciones.

\begin{tabular}{|lcc|}
\hline \multicolumn{1}{|c}{ Factor de análisis } & \multicolumn{2}{c|}{ Cantidad Porcentaje } \\
\hline \hline Producción y productividad & 22 & $44.0 \%$ \\
Competitividad & 4 & $8.0 \%$ \\
Niveles tecnológicos I+D+I & 6 & $12.0 \%$ \\
Oportunidad del sector & 13 & $26.0 \%$ \\
Talento humano & 5 & $10.0 \%$ \\
\hline
\end{tabular}

Para [55] la productividad es la forma de evaluar la eficiencia en el trabajo y el capital para realizar la generación de productos y servicios que tengan importe mercantil. Una mayor productividad está supeditada a que se está generando más importe mercantil con menos recursos, sin dejar de lado el cumplimiento de los requisitos exigidos para ellos. Su relación consiste en que se aumenta la fabricación sin tocar o aumentar los insumos para esta.

La concepción de competitividad parte de la dependencia atributo-coste, del precio de las materias primas utilizadas y de horizontes de sueldos en la nación que producen. Así también, directrices del gobierno [56] plantean que la productividad está inmersa en forma directa y proporcional a la competitividad. El Foro Económico Mundial (FEM) concibe la competitividad como la reunión de organizaciones, directrices gubernamentales y componentes que establecen el horizonte de una nación, siendo el principal factor para lograr más ingresos, desarrollo y prosperidad.

Para [57] se requiere hacer un examen duro. Al analizar las oportunidades del sector es primordial equiparar la ventaja competitiva con que se cuenta, identificar al comprador viable, plantear y ejecutar acciones con habilidades organizacionales, logrando que el producto o servicio que se oferta tenga una verdadera opción con posición conveniente en el mercado.

Según [58] ciencia, tecnología e innovación se requieren para lograr diferenciar y volver más sofisticado todo el sistema de producción, llegando a aumentar la eficiencia y eficacia de los mismos sistemas y de toda su dinámica económica. Esto logra mayor productividad y se obtiene un alto nivel de crecimiento.

El talento humano hace referencia a todos aquellos integrantes del sector productivo de forma directa e indirecta que aportan con su labor, sea operacional o intelectual, a la generación de productos y servicios. En el sector metalmecánico este factor cobra especial in- 
terés porque incide de forma esencial. [59] sostiene que el análisis del talento humano afecta de forma determinante el sector metalmecánico, al sustentar franjas reveladoras de fabricación donde hay alto uso de personal operativo por su aforo de empuje en el integrado de producción de los departamentos y su marca sobre la entrada económica y la prosperidad de la población.

El estudio muestra que el $44 \%$ de las investigaciones analizaron el factor de productividad y producción como elemento que genera empleo y valor agregado en la distribución regional. Sin embargo, señala que este sector requiere mejoras continuas a nivel organizacional y operacional, precisando que se realicen seguimientos a la producción desde una estrategia competitiva, que debe incluir investigación in situ con el objetivo de identificar la situación actual de la organización y cómo la estrategia le da valor agregado al proceso de producción. Debe tenerse en cuenta que la capacidad de producción de las empresas ha disminuido, pero la demanda ha aumentado en este sector, diferencia que se está cubriendo con las importaciones de acero de otros países. [60] propone plantear estrategias nacionales que permitan proteger la producción nacional, especialmente de aquellos productos que el país tiene capacidad de producir.

A su vez, el $8 \%$ analizó el factor competitividad, que debe incluir la acción con que cuenta una empresa para fabricar, proveer, dar nuevas alternativas de productos y servicios con calidad e importe. Lo anterior para dar acceso a un estadio superior en las actividades comerciales, logrando que los componentes que intervienen en el proceso obtengan un plus. Sin embargo, no se debe omitir la relación con la competitividad nacional que un país debe tener para la fabricación y ubicación de servicios y bienes en los comercios globales desde un puesto privilegiado, a partir de la calidad y correspondencia a las exigencias de dichos mercados.

El $12 \%$ de los estudios analizados consideraron el factor niveles tecnológicos como fundamental, determinando que el sector debe enfrentarse a tecnología de avanzada y centros de mecanizado de países como Corea y China. Además, debe concentrarse en manejar la situación con la competencia internacional, que ofrece productos más baratos a partir de una mano de obra más económica. Estos estudios señalan que el sector metalmecánico se ha convertido en un vehículo para dinamizar la economía de las regiones. Se considera importante que estas realicen nuevos desarrollos tecnológicos diferentes a las aeropartes. Estos desarrollos deben llevarlos a cabo no solo las grandes empresas, sino también los microempresarios del sector. Para este es importante el impulso en innovación y tecnología, en investigación y en acción conjunta con la certificación en gestión de la calidad, esto con el fin de poder fortalecer el sector desde las regiones.
El factor oportunidades del sector obtuvo un $26 \%$, en donde aparece la diversificación inteligente para la industria metalmecánica en Colombia. Allí presentan los caminos tecnológicos para alcanzar una mayor sofisticación a partir de las capacidades y conocimientos presentes en la industria metalmecánica. Los estudios proyectan la construcción de una ruta tecnológica que permita incrementar la complejidad del proceso, siguiendo la ruta tecnológica ya conocida con cierta cercanía en el manejo de materiales trabajados en este momento y brindando la posibilidad de nuevas oportunidades de diversificación de productos y sofisticación en la industria metalmecánica a partir de las capacidades existentes.

El talento humano fue analizado por el $10 \%$, mostrando las necesidades de capacitación del talento humano de las empresas del sector metalmecánico. También se analizan los niveles de cumplimiento de los diferentes factores de riesgo y las normas de competencia laboral. Este factor influye de manera notable en la competitividad del sector, pues el nivel de competencia hallado se cataloga como excelente en algunas regiones. Sin embargo, es necesario articular de manera eficiente la necesidad de capacitación del sector versus la oferta educativa, para que sea pertinente con respecto a las necesidades del sector a nivel profesional, técnico y tecnológico.

Así mismo, desde las universidades se pueden articular procesos de investigación e innovación para disminuir la poca transferencia de investigación a las empresas.

También se hallaron factores relacionados, es decir, factores que se encontraron inmersos y trabajados simultáneamente en un mismo estudio. Se obtuvo que productividad y competitividad fueron analizados en 25 estudios; producción y niveles tecnológicos en 23 e innovación y oportunidades del sector en 8 estudios. Lo anterior plantea que los factores analizados no son excluyentes, por el contrario, requieren de una alineación rigurosa que incluya una ruta tecnológica y estratégica para generar diversificación y sofisticación de los productos y de los procesos productivos.

\section{Conclusiones}

A partir de los resultados generales de la revisión documental se puede concluir que el sector metalmecánico en Colombia precisa de una alta competitividad si desea obtener permanencia y evolución. La dinámica del sector incluye entender los factores que más afectan su quehacer económico y operativo: productividad y producción, competitividad, niveles tecnológicos, oportunidades del sector y talento humano. Estos son los factores de producción de mayor análisis en los estudios utilizados en esta investigación, lo que permiten contextualizar el sector.

Para entender el sector metalmecánico en Colombia es necesaria la utilización de estrategias de disminución 
de costos, gestión de calidad, aplicación de forma valuada y significativa de la acumulación de experiencia y construcción de cadenas de valor en torno a estructuras de trabajo mancomunado, así como también la identificación de la brecha tecnológica para poder evitar el incumplimiento de los objetivos organizacionales en referencia directa a la rentabilidad. Esto con el fin de alcanzar una ubicación privilegiada en el ranking del mercado y lograr capacidad efectiva para competir y sobresalir.

\section{Referencias}

[1] J. M. Lesmes. (2018, Sep 21). Sector metalmecánico, uno de los más grandes del país. Radio SANTAFE. [Online]. Available: http://diariolaeconomia.com/ fabricas-e-inversiones/item/3913-sector-metalme canico-uno-de-los-mas-grandes-del-pais-fedemetal. html.

[2] R. Henao. (2017, Mar 13). Industria metalmecánica, a competir con sostenibilidad. Unimedios. Agencia de Noticias Universidad Nacional de Colombia. [Online]. Available: https://agenciadenoticias. unal.edu.co/detalle/article/industria-metalmecani ca-a-competir-con-sostenibilidad.html.

[3] PROCOLOMBIA. (s.f.). La Industrial Metalmecánica en COLOMBIA. Explore Oportunidades de Negocio. Industria Metalmecánica. [Online]. Available: http://www.procolombia.co/compradores/ es/explore-oportunidades/industria-metalmec-nica.

[4] H. J. Rubin and I. S. Rubin, El arte de escuchar datos. Thousand Oaks, CA: SAGE Publications, 2015.

[5] J. Ramírez, "Sector Metalmecánico del Departamento de Santander en El Periodo 2010-2015," presentado en el II Congreso Internacional en Administración de Negocios Internacionales, Bucaramanga, Colombia, 2017.

[6] S. Pérez and D. Zambrano, "Niveles tecnológicos de las empresas del sector metalmecánico en Boyacá desde el diseño y manufactura asistida por computador (CAD/CAM)," I3+, vol. 2, no. 2, pp. 114-132, Aug. 2015.

[7] A. Guzmán and C. Murcia, "La innovación tecnológica: mecanismo de competitividad para la creación de un clúster en el sector metalmecánico de los municipios de Cali y Yumbo," Revista Gestión \&3 Desarrollo, vol. 9, no. 1, pp. 27-35, Jan.Dec. 2015.

[8] G. Ovallos and S. Amar, "Perfil innovador de la industria manufacturera colombiana. Caso del sector Metalmecánico de Barranquilla," Revista Ingenierías, vol. 13, no. 25, pp. 115-136, Jul.Dec. 2015.

[9] E. Álzate and J. Marín and M. Tamayo, "Sector Metalmecánico de Risaralda un Diamante listo para ser pulido," Trabajo de grado. Facultad de ciencias Administrativas, Económicas y Financieras. Administración de Negocios Internacionales, Fundación Universitaria del Área Andina, Pereira, 2015.

[10] L. Daniels and A. Núñez, "Valoración de la Empresa Técnica Metalmecánica del Caribe y Cia. Ltda. Una Empresa del Sector Metalmecánico de la Ciudad de Cartagena," Trabajo de Grado Especialista. Facultad de Ciencias Económica. Universidad de Cartagena, Cartagena, 2015.

[11] M. Palacio, "Propuesta de implementación de sistema general de mantenimiento basado en mantenimiento productivo total y confiabilidad en el sector metalmecánico," Trabajo de grado Ingeniería. Industrial, Facultas de ingenierías, Universidad de San Buenaventura, Medellín, 2015.

[12] L. Ariza and M. Padilla, "Propuesta de mejoramiento de la productividad de una Pyme del sector metalmecánico de estructuras en Bogotá como estrategia para competir contra las importaciones de China," Trabajo de grado Ingeniería Industrial, Facultad de Ingeniería, Pontificia Universidad Javeriana, Bogotá, 2015.

[13] Z. Viancha, C. Becerra and S. Delgado. Estudio Prospectivo para el Sector Metalmecánico en Boyacá. Aplicación de la Metodología Cualitativa de Prospectiva Laboral. Programa de Las Naciones Unidas para el Desarrollo PNUD. Oficina Colombia, 2014.

[14] C. Betancur, J. Galvis, L. Gómez, and J. Pérez, "Carga Física en Trabajadores del Área de Acabados en Industria Metalmecánica," Revista Colombiana de Salud Ocupacional, vol. 5, no. 4, pp. 2326, 2015.

[15] S. Delgado, "Prospectiva Laboral para el Sector Metalmecánico en Boyacá," Revista Ingenio Magno, vol. 5, no. 1, pp. 137-145, 2015.

[16] O. Ocampo and L. Vargas, "Determinación de brechas estructurales en la integración de la Responsabilidad Social en empresas de la región Centro-Sur de Caldas. Caso de Estudio: Medianas empresas del sector metalmecánico," Revista ciencias Estratégicas, vol. 3, no. 1, pp. 60-71, Jun. 2016.

[17] A. Monroy and G. Puerta, "Metodología para implementar las 5's en empresas del sector metalmecánico del corredor industrial de Boyacá," I3+, vol. 2, no. 2, pp. 114-132, 2016.

[18] C. Carreño, M. González, and N. Patarroyo, "Diagnóstico del nivel de capacitación del personal vinculado al sector siderúrgico y metalmecánico de Boyacá respecto a las normas de competencia laboral," Revista Científica, vol. 4, no. 27, pp. 382-394, 2016.

[19] G. González and J. Ramírez, "Dinámica de la cadena priorizada del sector metalmecánico del 
departamento de Santander en el período 20102015," Revista Lebret., no. 8, pp. 175-199, 2016.

[20] Cede Trabajo, "La industria metalmecánica en Colombia frente a los TLC," Cede Trabajo. Informe SIA \# 23, Bogotá, Oct. 2016.

[21] D. López, "Factores de calidad que afectan la productividad y competitividad de las micros, pequeñas y medianas empresas del sector industrial metalmecánico," Revista Entre Ciencia e Ingeniería, vol. 10, no. 20, pp. 99-107, Sep. 2016.

[22] C. Rivera, "Diseño de un modelo para la implementación de procesos de innovación aplicables a la industria en empresas metalmecánicas en BOGOTÁ," Tesis de Maestría, Facultad de Ingeniería, Universidad Nacional de Colombia, Bogotá, 2016.

[23] M. Alba and A. Pinzón, "Una mirada retro y prospectiva en las Pymes del sector metalmecánico de Bogotá," Revista Vía Libre, vol. 9, no. 7, pp. 4461, 2016.

[24] A. Padilla and J. Rivera, "Sector autopartes en Colombia: comportamiento financiero durante el período 2008-2014," Revista Economía, vol. 12, no. 1, pp. 12-19, 2016.

[25] M. Alba and A. Pinzón, "Una mirada retro y prospectiva en las Pymes del sector metalmecánico de Bogotá," Revista Vía Libre, vol. 9, no. 7, pp. 44$61,2016$.

[26] Y. Burgos, E. Gómez, A. Pérez and J. Puello. "Diseño del modelo de planeación de operaciones de compras e inventarios aplicado al sector metalmecánico de la ciudad de CARTAGENA," presentada en 14 th LACCEI International MultiConference for Engineering, Education, and Technology: Engineering Innovations for Global Sustainability. San José, Costa Rica, 2016.

[27] D. Triana, "Elementos determinantes del proceso de desarrollo de nuevos productos en cinco pymes metalmecánicas: método hermenéutico fenomenológico," Tesis, Maestría en Administración, Facultad de Ciencias Económicas, Escuela de Administración y Contaduría Pública, Universidad Nacional de Colombia, Bogotá, 2016.

[28] G. González, J. Ramírez, and M. Vásquez, "Comercio intrarregional de los sectores autopartes y metalmecánico del departamento de Santander con los países miembro de la Alianza del Pacífico en el periodo 2012-2015," Revista Equidad Desarrollo, vol. 1, no. 28, pp. 113-146, 2017.

[29] EJE21. (2017, mar. 21). Industria metalmecánica, a competir con sostenibilidad. [Online]. Available: https:/ /www.eje21.com.co/2017/03/industria-metalmecanica-a-competir-con-sostenibilidad/.

[30] K. Salazar, "Análisis estratégico a partir del modelo de prácticas de gestión verde en las empresas industriales del sector metalmecánico de Maniza- les, Caldas Colombia," Tesis de Maestría, Facultad de Administración Departamento de Administración, Universidad Nacional de Colombia, Manizales, 2017.

[31] J. Castaño and K. Ríos, "Relaciones Comerciales entre los Países Miembros de la Alianza Del Pacífico En El Sector Metalmecánico," Revista Facultad de Ciencias Económicas, Administrativas y Contables, no. 7, pp. 113-129, 2017.

[32] J. Marín, J. Pascuas, and M. Rojas, "Inversión extranjera directa en el sector metalmecánica de Risaralda Colombia," Revista Cuaderno de Investigaciones, Semilleros Andina, vol. 9, no. 9, pp. 109-142, 2017.

[33] A. Vargas, "Diseño de un plan de gestión integral de residuos sólidos para una industria metalmecánica en la localidad de Puente Aranda (BogotáColombia)," Trabajo de grado Tecnología, Escuela de Ciencias Agrícolas, Pecuarias y del Medio Ambiente, Universidad Nacional Abierta y a Distancia, Bogotá, 2017.

[34] C. Acevedo, "Bienes de Capital desde el Caribe Colombiano, la historia de SUPERBRIX," Revista Dimensión Empresarial, vol. 15, no. 2, pp. 191204, 2017.

[35] A. Bernal and C. Beltrán, "Aplicación de herramientas Lean Manufacturing en los procesos de recepción y despacho de la empresa HLF Romero S.A.S," Trabajo de grado Maestría, Facultad de Ingeniería, Universidad de La Salle, Bogotá, 2017.

[36] B. Guerrero and C. Jaramillo, "Escenarios futuros del sector metalmecánico. Municipio de Tuluá y su zona de influencia," Revista Informador Técnico, vol. 82, no. 2, pp. 181-208, Nov. 2018.

[37] B. Alzate and S. Bernal, "Estrategias Lean Manufacturing para una empresa del sector metalmecánico," Trabajo de grado, Facultad de Ingenierías, Ingeniería Industrial, Universidad de San Buenaventura, Medellín, 2018.

[38] L. Baque, M. Herrera, and L. Mera, "Desarrollo De Estrategias De Manufactura Sostenible En La Micro, Pequeña Y Mediana Empresa Metal Mecánica (MIPYMES)," Journal of Alternative Perspectives in the Social Sciences, vol. 9, no. 3, pp. 547-554, 2018.

[39] C. Franco, E. Preciado, J. Rodríguez and J. Sáenz, "Diversificación Inteligente: Posibilidades de diversificación y sofisticación de la Industria Metalmecánica en Colombia," DATLAS Colombia. 2018. Available: https://www.bancoldex.com/sites/defa ult/files/documentos/perfil_industrial_metalmecanica.pdf.

[40] D. Triana, "Modelo para el diagnóstico de madurez en la Gestión De Proyectos para Mipymes de mantenimiento del sector metalmecánico en la localidad de KENNEDY". Trabajo de grado Maes- 
tría. Facultad de Ingeniería. Universidad Militar Nueva Granada, 2018.

[41] E. Arroyo, E. Perdomo, A. Quintero, and J. Rangel, "Análisis de los canales de distribución de las MIPYMES del sector metalmecánico de la ciudad de Barranquilla para establecer un modelo de negocio en el sector," Revista Investigación y Desarrollo en TIC, vol. 9, no. 1, pp. 48-54, 2018.

[42] E. Jassir, M. Domínguez, C. Paternina, and G. Fuentes, "Impacto de los indicadores del modelo SCOR para el mejoramiento de la cadena de suministro de una siderúrgica, basados en el ciclo cash to cash," Revista Innovar, vol. 28, no. 70, pp. 147-163, 2018.

[43] J. Escobar and I. Rodríguez, "Incidencia diferencial de los regímenes laborales Mipyme y común en el estado de resultados de las empresas del sector metalmecánico de la ciudad de Cajamarca para el año 2017," Universidad Privada del Norte, Perú, 2018.

[44] C. Giraldo, C. Guerrero and J. Marín, "Oportunidades que la alianza del pacífico crea en beneficio del sector metalmecánico a la competitividad de las pymes del municipio de Dosquebradas," Trabajo de grado, Administración de negocios internacionales, Facultad de Ciencias Administrativas, Económicas y Financieras, Fundación Universitaria del Área Andina, 2018.

[45] Enfoque Competitivo, "Enfoque competitivo- Negocio Metalmecánico," Cámara de Comercio de Cali. Grupo Estudios empresariales y de competitividad. Informe 109, 2018.

[46] Diario la Economía. (2018, Sep. 21). Sector metalmecánico, uno de los más grandes del país: Fedemetal. [Online]. Available: http://diariolaeconom ia.com/fabricas-e-inversiones/item/3913-sectormetalmecanico-uno-de-los-mas-grandes-del-paisfedemetal.html.

[47] R. Canabal, "Diseño de arquitectura de negocio basado en capacidades de tecnologías de la información para pyme del sector metalmecánico," Revista Teknos, vol. 18, no. 1, pp. 48-55, 2018.

[48] D. Sánchez, J. Aponte and J. torres. (2018, Dec. 1). Criterios de Implementación ISO 14001:2015 estudio de caso FUTECO Sector Metalmecánico. [Online]. Available: https://stadium.unad.edu.co/ preview/UNAD.php?url=/bitstream/10596/2318 3/1/jmaponteg.pdf.

[49] L. Hernández and M. Orjuela "Estudio de Caracterización de las Áreas de Mantenimiento en Empresas del Sector Metalmecánico en Bogotá y Sus Alrededores," Trabajo de Grado, Facultad Tecnológica, Universidad Distrital Francisco José de Caldas, Bogotá, 2018.

[50] J. Rodríguez, "Tendencia de la cultura organizacional en las pymes metalmecánicas en la local- idad de Fontibón," Trabajo de grado Maestría, Escuela de Ciencias Administrativas Económicas y de Negocios, Universidad Nacional Abierta y a Distancia, Bogotá D.C, 2018.

[51] A. Lache and J. Millán, "Rediseño del Sistema de Gestión de la Calidad a través de metodologías de mejora de procesos para incrementar la productividad en una empresa metalmecánica de Bogotá," Trabajo de grado Especialización, Escuela de Posgrados, Universidad Sergio Arboleda, Bogotá, 2018.

[52] K. Acosta and E. Muñoz, "Aspectos del plan de prevención, preparación y respuestas ante emergencias en el Sistema de Gestión de la Seguridad y Salud en el Trabajo," Trabajo de grado, Administrador de Empresas, Universidad Santo Tomás, Bogotá, 2018.

[53] N. Mercado, J. Meza, T. Obredor, and K. Salas, "Evaluación de la Cadena de Suministro para Mejorar la Competitividad y Productividad en el Sector Metalmecánico en Barranquilla, Colombia," Revista Información Tecnológica, vol. 30, no. 2, pp. 25-32, 2019.

[54] C. Pantoja, J. Orejuela, and J. Bravo, "Metodología de distribución de plantas en ambientes de agrupación celular," Revista Estudios gerenciales, vol. 33, no. 143, pp. 132-140, 2017.

[55] R. Padilla, C. Daneshvar, S. Garry, R. Gilbert, J. López, R. Minzer, J. Pérez, V. Quiroz, J. Rivas, I. Romero and J. Villarreal, "Productividad y brechas estructurales en México," Comisión Económica para América Latina y el Caribe, México, 2016.

[56] Consejo Privado de competitividad, "Informe Nacional de Competitividad 2018-2019," Bogotá, Colombia, 2018.

[57] E. Ortiz, M. Tamayo and J. Marín, "Sector metalmecánico de Risaralda un diamante listo para ser pulido," Trabajo de grado, Facultad de Ciencias Administrativas, Económicas y Financieras, Fundación Universitaria del Área Andina, Pereira, 2015.

[58] Consejo Privado de competitividad, "Informe Nacional de Competitividad 2018-2019," Bogotá, Colombia, 2018.

[59] R. Botía, "El Sector Metalmecánico: Perfiles laborales y oportunidades de inclusión social en el corredor Duitama-Sogamoso de Boyacá," Revista In Vestigium Ire, vol. 6, pp. 41-54, 2013.

[60] CedeTrabajo, "La industria metalmecánica en Colombia frente a los TLC," CedeTrabajo. Informe SIA 23, Oct. 2016. 